\title{
Recurrent Rearrangement of the PHF1 Gene in Ossifying Fibromyxoid Tumors
}

\author{
Samuel Gebre-Medhin, ${ }^{*}$ Karolin H. Nord, ${ }^{*}$ \\ Emely Möller, ${ }^{*}$ Nils Mandahl, ${ }^{*}$ Linda Magnusson, ${ }^{*}$ \\ Jenny Nilsson, ${ }^{*}$ Vickie Y. Jo, ${ }^{\dagger}$ \\ Fredrik Vult von Steyern, ${ }^{\ddagger}$ Otte Brosjö, ${ }^{\S}$ \\ Olle Larsson, " Henryk A. Domanski, ${ }^{\text {, }}$ Raf Sciot, ${ }^{\text {** }}$ \\ Maria Debiec-Rychter, ${ }^{\dagger+}$ Christopher D.M. Fletcher, ${ }^{\dagger}$ \\ and Fredrik Mertens*

\begin{abstract}
From the Department of Clinical Genetics, ${ }^{*}$ University and Regional Laboratories, and the Departments of Orthopedics ${ }^{\ddagger}$ and Pathology," Skåne University Hospital, Lund University, Lund, Sweden; the Department of Pathology, ${ }^{\dagger}$ Brigham and Women's Hospital, Harvard Medical School, Boston, Massachusetts; the

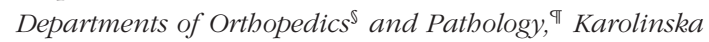
Hospital, Stockholm, Sweden; and the Department of Pathology,** Catholic University of Leuven and University Hospitals, and the Center for Human Genetics, ${ }^{+\dagger}$ Catholic University of Leuven,

Leuven, Belgium
\end{abstract}

Ossifying fibromyxoid tumor (OFMT) is a soft tissue tumor of unknown lineage. Although most cases are histologically and clinically benign, some show malignant morphological features and local recurrences are not uncommon; a few may even metastasize. In the present study, cytogenetic analysis identified different structural rearrangements of chromosome band 6 p21 in tumor cells from three cases of OFMT, including one with typical, one with atypical, and one with malignant morphological features. Mapping of the 6p21 breakpoint by fluorescence in situ hybridization (FISH) indicated that the PHF1 gene was rearranged in all three cases. Further FISH, 5'-rapid amplification of CDNA ends, and RT-PCR analyses disclosed an $E P 400 / P H F 1$ fusion transcript in one of the cases. Interphase FISH on tumor sections from 13 additional cases of OFMT showed rearrangement of the PHF1 locus in four of four typical, two of three atypical, and one of six malignant lesions. Thus, the PHF1 gene, previously shown to be the 3 -partner of fusion genes in endometrial stromal tumors, is also recurrently involved in the pathogenesis of OFMTs, irrespective of whether they are diagnosed as typical, atypical, or malignant lesions. The PHF1 protein interacts with the polycomb-repressive complex 2
(PRC2), which, in turn, regulates the expression of a variety of developmental genes. Thus, the results indicate that deregulation of PRC2 target genes is crucial for OFMT development. (Am J Pathol 2012, 181:10691077; http://dx.doi.org/10.1016/j.ajpath.2012.05.030)

Ossifying fibromyxoid tumor (OFMT) of soft parts is an uncommon tumor of uncertain cellular origin that predominantly occurs in the extremities of adults. ${ }^{1}$ Clinically, the tumor often presents as a small painless s.c. mass displaying a partial peripheral ring of calcification on radiography. Microscopically, there are often nests and cords of round-ovoid cells in a myxoid stroma surrounded by an incomplete layer of ossified tissue. However, up to $20 \%$ of the tumors lack apparent ossification and are termed nonossifying OFMT. ${ }^{2,3}$ Atypical and malignant examples of OFMT were reported in $1995,{ }^{4}$ but there has been some subsequent controversy regarding the aggressiveness of OFMT. Folpe and Weiss ${ }^{3}$ reviewed 70 cases, which they subdivided into typical, atypical, and malignant OFMT on the basis of nuclear grade, cellularity, and mitotic rate. Local recurrences and metastases were seen in all three subtypes, but more often among the malignant lesions (60\% recurred locally, and $60 \%$ metastasized) than among the typical (12\% and $4 \%$, respectively) and atypical (13\% and $6 \%$, respectively) lesions. Miettinen et al, ${ }^{5}$ reviewing 104 cases, also noted a correlation between mitotic rate and local recurrence, but questioned the existence of malignant, metastasizing OFMT; according to them, such lesions represented misdiagnoses. A recent study by Graham et al, ${ }^{6}$ using immunohistochemistry (IHC) and gene expression profiling, showed that cases classified as typical show extensive overlap with those classified as malignant.

Supported by The Swedish Cancer Foundation, the National Research Council of Sweden, the Gunnar Nilsson Cancer Foundation, the IngaBritt and Arne Lundberg Foundation, and the Medical Faculty of Lund University.

Accepted for publication May 30, 2012.

Supplemental material for this article can be found at http://ajp. amjpathol.org or at http://dx.doi.org/10.1016/j.ajpath.2012.05.030.

Address reprint requests to Samuel Gebre-Medhin, M.D., Ph.D., Department of Clinical Genetics, University Hospital, SE-221 85 Lund, Sweden. E-mail: samuel.gebre-medhin@med.lu.se. 
1070 Gebre-Medhin et al

AJP September 2012, Vol. 181, No. 3

Table 1. Clinicopathologic Features of 17 Patients with OFMT

\begin{tabular}{|c|c|c|c|c|c|c|}
\hline $\begin{array}{l}\text { Patient } \\
\text { no. }\end{array}$ & $\begin{array}{c}\text { Age } \\
\text { (years)/sex }\end{array}$ & $\begin{array}{l}\text { Primary site } \\
(\text { size })^{\star}\end{array}$ & Diagnosis & Relapse time (years) & $\begin{array}{l}\mathrm{FISH} \text { for } \\
\mathrm{PHF1}^{+}\end{array}$ & $\begin{array}{l}\text { FISH for } \\
\text { SMARCB1 }\end{array}$ \\
\hline 1 & $59 / \mathrm{M}$ & Shoulder $(N A)^{\S}$ & Typical & $\begin{array}{l}\text { LR: } 7,10,12,13,19 \\
\text { MT: } 19{\text { (leg })^{\S}}^{\text {leg }}\end{array}$ & Split? & ND \\
\hline 2 & $47 / F$ & Temple (1.5) & Typical & & Split & 2 Copies \\
\hline 3 & $50 / \mathrm{M}$ & Foot (4.5) & Typical & & Split & 2 Copies \\
\hline 4 & $41 / F$ & Back (5) & Typical & & Del 5' & 2 Copies \\
\hline 5 & $76 / F$ & Shoulder (NA) & Typical & & Del 5' & 2 Copies \\
\hline 6 & $71 / \mathrm{M}$ & Thigh (10) & Atypical & & Split & ND \\
\hline 7 & $47 / F$ & $\operatorname{Leg}(2)$ & Atypical & & Failure & Failure \\
\hline 8 & $53 / \mathrm{M}$ & $\operatorname{Hip}(N A)$ & Atypical & LR: $6^{\S}$ & Split & 2 Copies \\
\hline 9 & $71 / F$ & Popliteal fossa (3) & Atypical & & Del 5' & 2 Copies \\
\hline 10 & $59 / \mathrm{M}$ & $\operatorname{Neck}(N A)$ & Atypical & MT: 17 (rib), 19 (clavicle) $)^{\S}$ & NR & 2 Copies \\
\hline 11 & $63 / \mathrm{M}$ & Toe (3) & Malignant & LR: $2,4,6, \$ 7$ & Split? & ND \\
\hline 12 & $29 / F$ & Pelvis (7) & Malignant & MT: $3,5^{\S}$ (peritoneal) & Split & 1 Copy \\
\hline 13 & $42 / \mathrm{M}$ & $\operatorname{Neck}(6)$ & Malignant & & NR & 1 Copy? \\
\hline 14 & $43 / \mathrm{M}$ & Paralaryngeal (5) & Malignant & & NR & 1 Copy \\
\hline 15 & $82 / \mathrm{M}$ & Foot (5.5) & Malignant & & NR & 1 Copy \\
\hline 16 & $70 / F$ & Right leg (5) & Malignant & MT: 2 (left leg) $)^{\S}$ & NR & 1 Copy \\
\hline 17 & 70/F & Abdominal wall (4) & Malignant & & NR & Failure \\
\hline
\end{tabular}

Open spaces indicate absence of relapse.

* Largest diameter (in centimeters)

${ }^{+}$Del $5^{\prime}$, loss of the signal mapping the $5^{\prime}$ of PHF1 in $\geq 25 \%$ of the nuclei; Split, split signals for the probes mapping $5^{\prime}$ and $3^{\prime}$ of PHF1 in $\geq 25 \%$ of the nuclei. FTwo copies, two copies of $22 \mathrm{q}$ in $\geq 60 \%$ of the cells; 1 copy, one copy of $22 \mathrm{q}$ in $\geq 60 \%$ of the cells; 1 copy?, one copy of $22 \mathrm{q}$ in $30 \%$ of the cells.

$\S$ Material reviewed and analyzed in the instance of multiple lesions.

"Results from metaphase FISH. All other cases were analyzed by interphase FISH.

F, female; M, male; LR, local recurrence; MT, metastasis; NA, not available; ND, not done; NR, not rearranged.

The genetic information on OFMT is limited. Graham et $\mathrm{al}^{6}$ identified loss of chromosome 22 in five of seven cases analyzed by interphase fluorescence in situ hybridization (FISH). Among the three cases with abnormal karyotype published, no recurrent translocation has been found. ${ }^{7-9}$ In the present study, we analyzed three cases by chromosome banding analysis. Because all of them displayed structural rearrangements with a breakpoint in $6 \mathrm{p} 21$, a feature found also in one of the previously published cases, ${ }^{8}$ we considered it worthwhile to map the breakpoints by FISH. When finding that all 6p21 rearrangements potentially disrupted the PHF1 gene (encoding the PHD finger protein 1), previously shown to be the $3^{\prime}$-partner in fusion genes in endometrial stromal cell tumors (ESTs), ${ }^{10}$ further analyses using $5^{\prime}$-rapid amplification of cDNA ends (5'-RACE) PCR and RT-PCR were per- formed to disclose potential fusion gene transcripts. Finally, we analyzed the status of the PHF1 gene and chromosome 22 in tumor sections from additional OFMTs, representing typical, atypical, and malignant lesions.

\section{Materials and Methods}

\section{Patients}

The study included 17 patients with OFMT (9 men and 8 women; age range, 29 to 82 years; Table 1). From three of them (patients 1, 6, and 11), fresh and frozen tumor biopsy specimens were available for cytogenetic and molecular genetic analyses (Table 2). From the remaining 14 patients,

Table 2. Cytogenetic, Molecular Cytogenetic, and Molecular Genetic Data on Three Patients with OFMT

\begin{tabular}{|c|c|c|c|c|}
\hline $\begin{array}{l}\text { Patient } \\
\text { no. }\end{array}$ & Karyotype $^{*}$ & SNP array ${ }^{\dagger}$ & $\mathrm{FISH}^{\ddagger}$ & $\mathrm{PCR}^{\S}$ \\
\hline 1 & $\begin{array}{l}\text { LR: 19: 46,Y,ins(X;6)(p11;p21p25),t(2;12)(q31;q22); } \\
\text { MT: 47,Y,ins(X;6)(p11;p21p25),t(2;12)(q31;q22),+12 }\end{array}$ & $\begin{array}{l}\mathrm{ND} \\
\mathrm{G}: 9 \mathrm{q}, 12 ; \mathrm{L}: \\
\quad 7 \mathrm{q}, 8 \mathrm{p}\end{array}$ & $\begin{array}{l}\text { ND } \\
\text { PHF1 pos }\end{array}$ & $\begin{array}{l}\text { ND } \\
\text { RACE neg }\end{array}$ \\
\hline 6 & $\begin{array}{r}\text { 44,XY,6,der(7)inv(7)(p?11q22)ins (7;6)(q?22;p21p2?5) } \\
\text { t(6;12)(p21;q24),der(12)ins(12;6)(q24;p21p1?1),-13 }\end{array}$ & $\begin{array}{l}\text { L: } 6 \mathrm{q}, 7 \mathrm{p}, 9 \mathrm{q} \\
12 \mathrm{q}, 13\end{array}$ & $\begin{array}{l}\text { EP400 pos and PHF1 } \\
\text { pos }\end{array}$ & EP400/PHF1 \\
\hline 11 & $\begin{array}{l}\text { 45,Y, add }(\mathrm{X})(\mathrm{p} 11), \operatorname{del}(1)(\mathrm{p} 35), \operatorname{der}(3) \mathrm{t}(3 ; 16)(\mathrm{p} 12 ; \mathrm{p} 11), \\
\text { der(6)t(6;7)(p21;p15), der(7)t(X;7)(p2?;p15)t(X;6)(p11; } \\
\text { p21), del(13)(q?21), der(17)t(?13;17)(q2?2;q2?5),20 }\end{array}$ & ND & PHF1 pos & RACE neg \\
\hline
\end{tabular}

For more detailed information on detected imbalances, see Supplemental Table S3 (available at http://ajp.amjpathol.org).

* Rearrangements involving the PHF1 locus in 6p21 are boldfaced. In patient 11, the same karyotype was found in the primary lesion and in three relapses, spanning 4.5 years.

tChromosomes and chromosome arms affected by imbalances, as detected by SNP array analysis.

${ }^{\ddagger}$ Metaphase FISH for PHF1 and partner genes involved in potential fusions.

§Results of RT-PCR and 5'-RACE-PCR analyses. PCR

G, gains; L, losses; LR, local recurrence; MT, metastasis; ND, not done; neg, negative; pos, positive; RACE neg, no fusion transcript detected at 5'-RACE 
tumor sections were retrieved from the consultation files of one of the authors (C.D.M.F.).

\section{Histopathological Review}

H\&E-stained sections and IHC stains were re-examined. Tumors from five patients were classified as typical, five were classified as atypical, and seven were classified as malignant OFMT (Table 1). Tumors were designated as typical, atypical, or malignant, based on morphological features alone (see Results). From five patients, the primary tumor was unavailable for review, with only local recurrences being reviewed for patients 8 and 11 and distant metastases being reviewed for patients 10, 12, and 16 . From patient 1, both the primary lesion and the metastasis were analyzed.

\section{IHC Data}

IHC was performed at diagnosis at Brigham and Women's Hospital (Boston, MA) on sections ( $4 \mu \mathrm{m}$ thick) of formalin-fixed, paraffin-embedded tissue, using the following antibodies and conditions: epithelial membrane antigen (EMA; Dako, Carpentaria, CA; clone E29; 1:200 dilution, no pretreatment), S100 protein (Dako; polyclonal; 1:3000; no pretreatment), pan-keratin (Dako; clone MNF116; 1:700; 10-minute protease digestion), keratin AE1/AE3 (Dako; AE1+AE3; 1:200; 10-minute protease digestion), glial fibrillary acidic protein (GFAP; Dako; polyclonal; 1:15,000; citrate buffer and pressure cooker), desmin (Sigma, St Louis, MO; clone DE-U-10; 1:5000; citrate buffer and pressure cooker), and INI-1 (BD Biosciences, San Jose, CA; clone 25; 1:200; EDTA and pressure cooker). The Envision Plus detection system (Dako) was used for all antibodies. Appropriate positive and negative controls were used throughout.

\section{Cytogenetic and FISH Analyses}

\section{Chromosome Banding Analysis}

Cell culturing, harvesting, and G-banding were performed as described, and the karyotypes were written following the recommendations of the International System for Human Cytogenetic Nomenclature. ${ }^{11,12}$

\section{Metaphase FISH}

Metaphase spreads for FISH analysis were available from the same tumors that had been analyzed cytogenetically, except for the local recurrence in patient 1 (Table 2). The bacterial artificial chromosome (BAC) and fosmid probes that were used to delineate the breakpoints in 6p21 and on partner chromosomes were obtained from the BACPAC Resource Center (http:/bacpac. chori.org, last accessed February 2012; see also Supplemental Table S1 at http://ajp.amjpathol.org). The $\mathrm{BAC}$ and fosmid clones were selected based on their location in the National Center for Biotechnology Information Map Viewer (http://www.ncbi.n/m.nih.gov/projects/ mapview, build 36.3, last accessed February 2012) and the University of California, Santa Cruz, Human Genome Browser Gateway (http://genome.ucsc.edu/cgi-bin/hg Gateway, last accessed February 2012). To identify unequivocally tumor cells, whole chromosome painting probes (Applied Spectral Imaging, Migdal Haemek, Israel) were used. Clone preparation, hybridization, and analysis were performed as previously described. ${ }^{13}$

\section{Interphase FISH on Tumor Sections}

To search for rearrangements involving the PHF1 locus in additional tumors, cut sections ( $4 \mu \mathrm{m}$ thick) from paraffin-embedded tumor tissue from an additional 14 patients were retrieved and subjected to interphase FISH, with two BAC probes flanking the gene (see Supplemental Table S1 at $h$ ttp://ajp.amjpathol.org). Deletions affecting chromosome 22 were investigated with the LSI TUPLE1/LSI ARSA probe mix (Vysis, Downers Grove, IL), hybridizing to subbands 22q11.2 and 22q13.3. Hybridization and analysis were performed as previously described. ${ }^{13}$

\section{SNP Array Analysis and Data Interpretation}

DNA from two OFMTs (patients 1 and 6) was analyzed with single-nucleotide polymorphism (SNP) array to detect global copy number aberrations. The DNA was extracted from fresh frozen tumor biopsy specimens using the DNeasy Tissue Kit, including the optional RNaseH treatment, according to the manufacturer's instructions (Qiagen, Valencia, CA). DNA concentrations were measured with a Nanodrop ND-1000 spectrophotometer (Saveen \& Werner AB, Malmö, Sweden). DNA was hybridized to the Illumina HumanCNV370 Genotyping BeadChips (Illumina, San Diego, CA), following standard protocols supplied by the manufacturer. Data analysis was performed using the BeadStudio software version 3.1 (Illumina), and imbalances were identified through visual inspection.

\section{PCR Analyses}

From patients 1, 6, and 11, total RNA was extracted from frozen tumor tissue using the RNeasy lipid tissue kit, according to the manufacturer's instructions (Qiagen). Concentrations were measured as previously described, and the quality was assessed by using a 2100 Bioanalyzer (Agilent Technologies, Santa Clara, CA). The cDNA synthesis, 5'-RACE PCR, and nested PCR were performed using the SMARTer RACE cDNA Amplification kit, according to the manufacturer's instructions (Clontech, Mountain View, CA). The primers used for $5^{\prime}$-RACE PCR are listed in Supplemental Table S2 (available at $h t t p: / /$ ajp.amjpathol.org). Primers specific for PHF1, EP400, $F A M 188 B$, and $A Q P 1$ were designed to search for fusion transcripts using RT-PCR (Table 3). RT-PCR was performed as previously described. ${ }^{13}$ Transcripts were amplified using an initial denaturation for 5 minutes at $95^{\circ} \mathrm{C}$; followed by 35 cycles of 1 minute at $95^{\circ} \mathrm{C}, 1$ minute at $55^{\circ} \mathrm{C}$, and 1 minute at $72^{\circ} \mathrm{C}$; and a final extension for 10 minutes at $72^{\circ} \mathrm{C}$. Amplified fragments were purified from agarose gels and directly sequenced using the Big Dye version 1.1 cycle sequencing kit (Applied Biosystems, 
Table 3. Primers Used for $5^{\prime}$-RACE PCR and RT-PCR

\begin{tabular}{|c|c|}
\hline Primer name & Primer sequence \\
\hline AQP1-130F & 5'-AGGCCTCTCCAGCTTCTAGG-3' \\
\hline AQP1-361F & 5'-ATTAACCCTGCTCGGTCCTT- $3^{\prime}$ \\
\hline FAM-117F & 5'-GTCAGGGAGTTCCTCAGCAG-3' \\
\hline FAM-396F & 5' - GTGCCATCAAGATGCTCAGA-3' \\
\hline FAM-802F & $5^{\prime}-$ AAGAAGAGAGCCGGAAGGTC- $3^{\prime}$ \\
\hline FAM188B-1277F & $5^{\prime}$-GCAGACAGCATCAAAACCAA- $3^{\prime}$ \\
\hline FAM188B-1638F & $5^{\prime}$-CAGCAGTTCAGTCCAACAGG-3' \\
\hline FAM188B-1925F & 5'-CAACGTTTTCAACGATGTGG-3' \\
\hline EP400-6549F & $5^{\prime}$-ACAGTGAGGACGCAGTGATG-3' \\
\hline EP400-6599F & $5^{\prime}-$ CTGAAGACCCTGCAGGAGAG- $3^{\prime}$ \\
\hline PHF1-250R & 5'-AAGCTGGGTCCCAAAGTGAGGAGG-3' \\
\hline PHF1-250R short & 5'-AGCTGGGTCCCAAAGTGAGG-3' \\
\hline PHF1-327R & $5^{\prime}$-AGCCCATCAGTCCATCTGGCCAG-3' \\
\hline PHF1-327R short & 5'-AGCCCATCAGTCCATCTGGC-3' \\
\hline PHF1-426R & 5'-GGACCAGACACACCTCCCTA-3' \\
\hline PHF1-518R & $5^{\prime}$-GCGACAGACACAACAGAGGA-3' \\
\hline
\end{tabular}

Foster City, CA) on an ABI-3100 Avant genetic analyzer (Applied Biosystems). The BLASTN software (http://www. ncbi.n/m.nih.gov/blast, last accessed February 2012) was used for the analysis of PHF1 and EP400 sequence data.

\section{Results}

\section{Histopathological Features}

A total of 18 tumors from 17 patients (from patient 1 , both the primary lesion and a metastasis were studied) were reviewed by two of the authors (V.Y.J. and C.D.M.F.). All tumors demonstrated at least focal areas of typical OFMT, with reticular and cord-like growth of round-toovoid tumor cells characterized by uniformly sized vesicular nuclei embedded in a fibromyxoid background. Most tumors showed a multilobular growth pattern and at least partial encapsulation by a thick fibrous shell, some with characteristic ossification. In general, cytologic atypia ranged from minimal to none, and was observed to be moderate at most (notably in atypical and malignant cases). Tumors showing increased cellularity, at least focal nuclear atypia, more prominent mitotic activity, and bone formation within tumor lobules (whether present in fibrous septa) were classified as malignant; tumors showing only a subset of these features were labeled atypical. Figure 1, A-H, shows representative images of typical (patients 2 and 4), atypical (patient 9), and malignant (patient 16) tumors.

IHC staining was performed on one tumor from each patient (from patient 1 , only the primary lesion was analyzed). Overall, of 17 tumors, 9 were positive for S100 (2 focal) and 14 were positive for desmin (1 focal) (Figure 1, $\mathrm{C}$ and D). INI-1 (SMARCB1) staining was positive (ie, normal/retained), albeit sometimes weakly, in 13 of 13 tumors examined (see Supplemental Figure $\mathrm{S} 1$ at $h t t p: / /$ ajp.amjpathol.org). IHC staining results for all cases are summarized in Supplemental Table S2 (available at http://ajp.amjpathol.org).

Five patients were diagnosed as having typical OFMT. Two showed metaplastic bone in the capsule, and three were nonossifying. Tumors were composed of monomor- phic round-to-ovoid cells, frequently showing trabecular and cord-like growth patterns. Mitotic counts ranged from zero to seven per 10 high-power fields (median count, 1); three tumors showed no mitoses. Both the primary and metastatic lesions from patient 1 showed mild nuclear atypia and focally increased hypercellularity, whereas the other four lesions were entirely bland. S100 protein staining was positive in four tumors and negative in one tumor; desmin staining was positive in four of five tumors. No keratin (0/5), EMA (0/4), or GFAP (0/5) staining was observed. SMARCB1 (INI-1) staining was positive (ie, nor$\mathrm{mal} /$ retained) in three of three tumors.

Five tumors from five patients were classified as atypical OFMT, including a recurrent hip tumor (patient 8) and a metastasis to the clavicle from a neck primary tumor (patient 10). Four tumors were ossifying; in all instances, ossification was present between tumor cells within lobules. One tumor showed no evident ossification. Nuclear atypia ranged from negligible to moderate, with occasional hyperchromatic nuclei. All tumors showed at least focal hypercellularity with overlapping nuclei. Mitotic
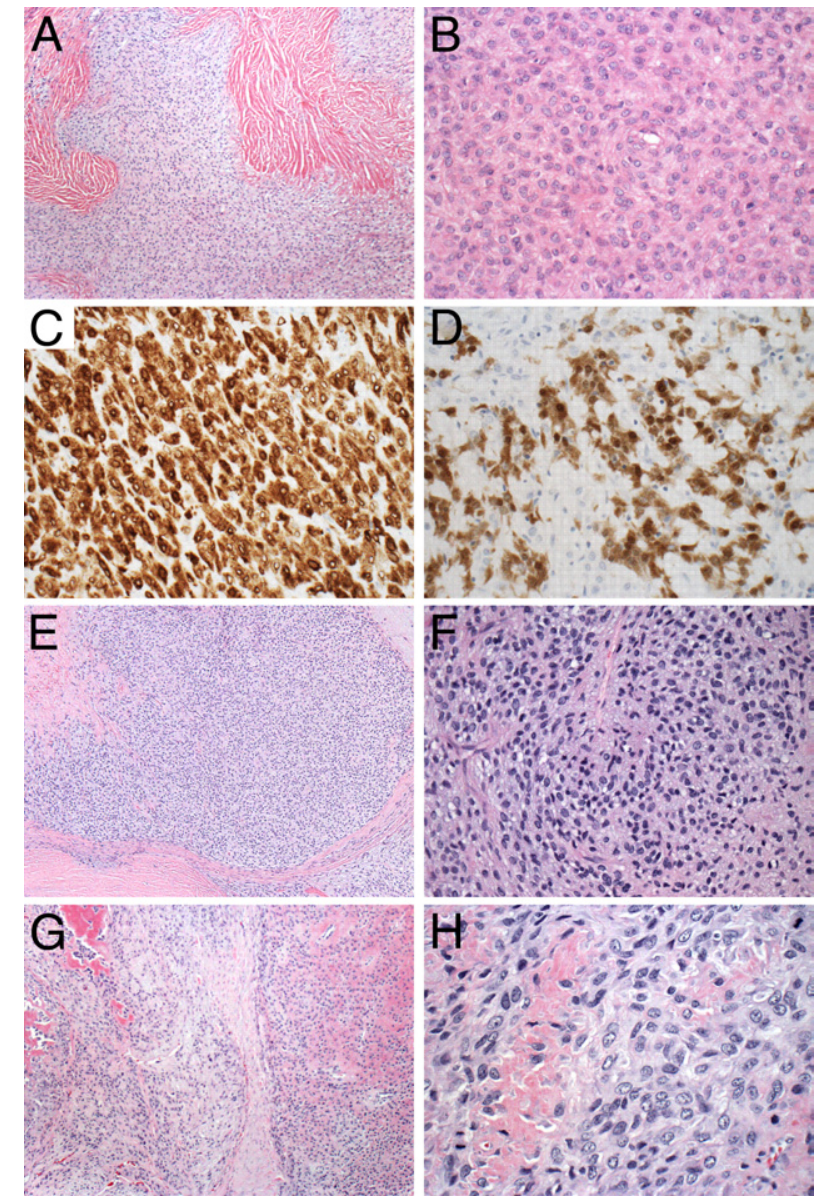

Figure 1. Morphological and IHC findings in OFMTs. Typical OFMT (patient 4) showing multilobular growth (A) and ovoid-to-round cells embedded in fibromyxoid stroma (B). Immunoreactivity for desmin (C) and nuclear staining for S100 (D) (patient 2) are observed in a subset of tumors. E: Atypical OFMT (patient 9) showing increased cellularity. F: Mild atypia and hyperchromatic nuclei. G: Ossification within tumor nodules and increased cellularity in a case of malignant OFMT (patient 16), with overlapping hyperchromatic nuclei showing mild atypia $(\mathbf{H})$. Original magnification: $\times 10(\mathbf{A}, \mathbf{E}$, and G); $\times 40(\mathbf{B}-\mathbf{D}, \mathbf{F}$, and $\mathbf{H})$. 
counts ranged from 8 to 29 per 10 high-power fields (median count, 10). Three of five tumors were positive for S100 protein. Desmin staining was diffusely positive in three tumors, focal in one tumor, and negative in one tumor. No immunoreactivity was observed for keratin (0/2), GFAP (0/2), and EMA (0/3). SMARCB1 staining was positive in four of four tumors.

Seven patients were diagnosed as having malignant OFMT, including a locally recurrent toe tumor (patient 11), a peritoneal metastasis from a pelvic primary tumor (patient 12), and a metastatic left leg lesion from a right leg primary tumor (patient 16). Four tumors were ossifying and showed ossification within the tumor lobules; three lacked ossification in the sections examined. All tumors showed areas of hypercellularity, with overlapping nuclei and frequently solid growth, in addition to areas with fibromyxoid stroma. Although all tumors demonstrated nuclear atypia and a pleomorphism, the degree ranged from mild to moderate at most. Some tumors showed focal areas of more spindled morphological features. Mitotic counts ranged from 8 to 27 per 10 highpower fields (median count, 16). S100 protein immunostaining was positive in two tumors, focal in one tumor, and negative in four tumors. Desmin staining was present in six of seven tumors. Keratin staining was focal in two of five tumors, and focal GFAP positivity was seen in one of four tumors. Focal EMA staining was seen in three of six tumors. SMARCB1 staining was positive in six of six tumors (see Supplemental Figure S1 at http://ajp.amjpathol.org).

\section{Cytogenetic Findings}

Tumors from patients 1,6 , and 11 were characterized cytogenetically after short-term culturing. All had neardiploid karyotypes with different rearrangements of $6 \mathrm{p} 21$ (Table 2). From patient 1, both a local recurrence and a metastasis could be analyzed, revealing identical karyotypes, except for the presence of trisomy 12 in the metastasis. Apart from 6p21, no other chromosome band was recurrently involved, but two tumors each had breakpoints in $7 p$ and $12 q$.

\section{Metaphase FISH Mapping of Breakpoints in 6p21, 12q24, and 7p15}

\section{Mapping of Breakpoints in 6p21}

The breakpoints in 6p21 in patients 1, 6, and 11 were mapped with a panel of whole chromosome painting, $\mathrm{BAC}$, and fosmid probes (probes delineating breakpoints are listed in Supplemental Table S1 at $h$ ttp://ajp. amjpathol. org). In all three patients, FISH using whole chromosome painting probes showed that none of the rearrangements affecting $6 p$ was a standard translocation; in two of them, there was an insertion of $6 p$ into Xp (patient 1) or $7 q$ and $12 q$ (patient 6), and in one (patient 11), $6 p$ was involved in a three-way exchange with chromosomes $X$ and 7 . In patient 1 , the $6 \mathrm{p} 21$ breakpoint in the ins $(X ; 6)$ was delineated by fosmid W12-1201P10, mapping to the der $(X)$, and BAC RP11-175A4, mapping to the der(6) (Figure 2). The $6 p$ rearrangement was more complex in patient 6 ; the der(7) contained material from 6p21-25, but not in a linear manner, and the der(12) carried an inverse insertion of 6p1?1-p21 in band 12q24. The breakpoint in 6p21 was delineated by fosmid W12-1617B20, mapping to the der(12), and BAC RP11-175A4, mapping to the der(7); the sequence recognized by fosmid W12-1201P10, mapping $5^{\prime}$ of PHF1, was only present on the normal chromosome 6 homologue and, thus, hemizygously deleted from the rearranged chromosome 6 . In patient 11, the 6 p21 breakpoint was delineated by BAC CTD-2307H19, which gave split signals on the $\operatorname{der}(7)$ and the $\operatorname{der}(6)$; the $3^{\prime}$ part of the PHF1 locus mapped to the $\operatorname{der}(6) t(6 ; 7)$, on which RP11-175A4 gave a signal (Figure 2A). Bearing in mind the resolution of metaphase FISH, a minimal shared breakpoint region in 6p21 could be mapped to position 33.43 to $33.49 \mathrm{Mb}$; this region contains three genes (LYPLA2P1, KIFC1, and PHF1).

The breakpoints in distal $6 p$ in the ins $(X ; 6)$ in patient 1 and the ins $(12 ; 6)$ in patient 6 differed by several megabases, thus excluding a second shared breakpoint in $6 p$ (data not shown).

\section{Mapping of Breakpoints Potentially Involved in Exchanges with 6p21}

Considering the transcriptional orientation of PHF1 (transcribed from telomere to centromere) and the FISH mapping of the 6p21 rearrangements, potential candidates for $5^{\prime}$ partners in fusions with the PHF1 locus should be located in 6p25 in patient 1, 12q24 in patient 6, and 7p15 in patient 11. The breakpoint in 6p25 in patient 1 was delineated by RP3-406P24, which gave split signals on the der(6) and the der(X); the segment covered by this BAC does not contain any known gene. The breakpoint in 12q24 in patient 6 was delineated by RP11-417L19, mapping to the 5' part of EP400; cohybridization of RP11417L19 and W12-2752E10 (covering PHF1) revealed a fusion signal in the der(7) (Figure 2B). The breakpoint in 7p15 in patient 11 was delineated by BAC RP11-902N23, which gave split signals on the der(6) and the $\operatorname{der}(7)$; this segment contains two genes (FAM188B and AQP1).

\section{Interphase FISH Analysis of Paraffin-Embedded Tumors}

Interphase FISH using BACs flanking the $5^{\prime}$ (CTD3221B10) and $3^{\prime}$ (CTD-2592B23) parts of PHF1 was successfully performed on cut tumor sections from 13 of 14 additional patients (Table 1). Rearrangement of the PHF1 locus (defined as aberrant signals in at least $25 \%$ of the nuclei) was seen in all four typical tumors (two with split signals and two with loss of the signal flanking the $5^{\prime}$ part of the gene), in two of three atypical tumors (one with split signals and one with loss of the signal flanking the $5^{\prime}$ part of the gene), and in one of six (split signal) malignant tumors (Figure 2C). The same 14 tumors were also analyzed with regard to deletions on chromosome 22 (Table 1). Of 12 successfully analyzed tumors, zero of four typical, zero of three atypical, and four of five malignant lesions showed loss of one copy of chromosome 22 (Fig- 

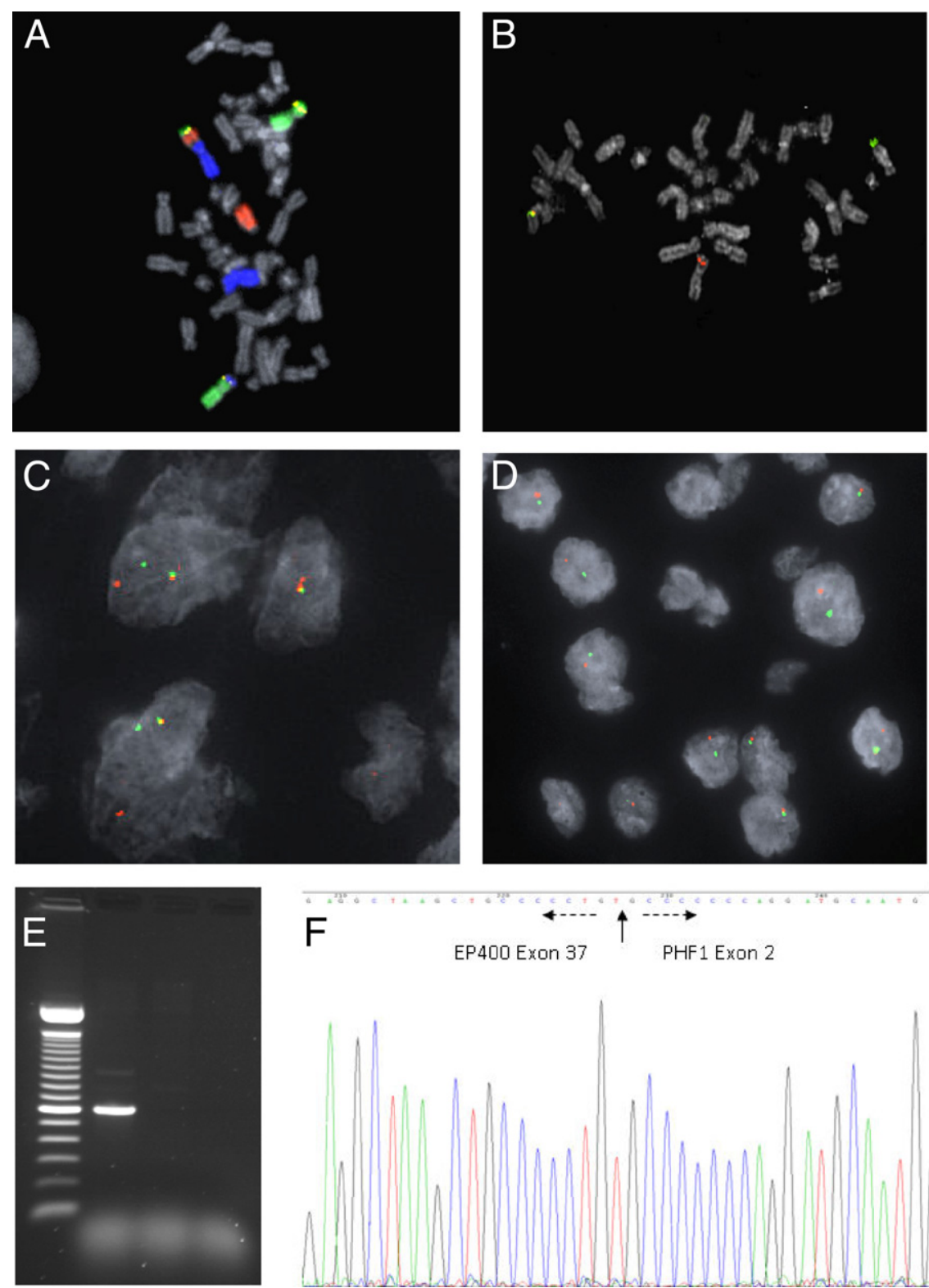

Figure 2. FISH and PCR analyses of ossifying fibromyxoid tumors. A: Complex exchange of material between chromosomes $\mathrm{X}$ (red), 6 (green), and 7 (blue) in patient 11; the BAC probe covering PHF1 (yellow) shows split signals on the $\operatorname{der}(6)$ and the $\operatorname{der}(7)$. B: In patient 6 , cohybridization of fosmid W12-2752E10 (red; PHF1) and BAC RP11-417L19 (green, 5' of EP400) showing a fusion signal (yellow) on the der(7). C: Interphase FISH with probes (CTD3221B10, green, $5^{\prime}$ of PHF1 and CTD-2592B23, red, $3^{\prime}$ of PHF1) flanking the PHF1 locus in case 8 , revealing split signals. D: Interphase FISH on a section from the tumor of patient 16, with probes for chromosome bands 22q11 (red) and 22q13 (green), reveals loss of one copy of chromosome 22. E: RT-PCR with primers specific for the EP4OO and PHF1 genes amplifies an approximately 600-bp fragment in patient 6 (lane 2), but not in patient 1 (lane 3). Lane 1, 100-bp ladder lane 4 , blank. F: Sequencing of the amplified transcript in patient 6 reveals an in-frame fusion of exon 37 of EP4OO with exon 2 of PHF1. ure 2D). The fifth malignant tumor was potentially positive (30\% of the nuclei showed hemizygous loss). One atypical tumor (patient 10) was negative for both PHF1 and chromosome 22 rearrangements, whereas one malignant tumor (patient 12) was positive for both rearrangements.

\section{SNP Array Results}

Both tumors (a morphologically benign lesion from patient 1 and a morphologically atypical lesion from patient 6) analyzed by SNP array showed chromosomal imbalances; no rearrangements affecting the PHF1 locus in 6 p21 or chromosome 22 were seen. In patient 6 , the detected imbalances included a deletion of the 3' part of the EP400 gene (Table 2; see also Supplemental Table S3 at $h$ ttp://ajp.amjpathol.org).

\section{PCR Findings}

5 '-RACE PCR did not result in any fragment in patients 1 and 11, but it amplified an approximately 750-bp fragment in patient 6 . Sequence analysis showed that the fragment was a hybrid cDNA product in which nucleotide 6846 of the EP400 gene (NM_015409), Iocated in chromosome band 12q24, was fused to nucleotide 221 of PHF1 (NM_024165). Subsequently, RTPCR with the primer combination EP400-6549F and PHF1-518R amplified an approximately 600-bp cDNA fragment (Figure 2E). Direct sequencing of the fragment disclosed an in-frame EP400/PHF1 chimeric transcript, in which exon 37 of EP400 was fused with exon 2 of PHF1 (Figure 2F).

Repeated RT-PCR analyses with various combinations of forward primers for the FAM188B and AQP1 genes and 
reverse primers for PHF1 did not result in any FAM188B/ PHF1 or AQP1/PHF1 fusion transcripts in patient 11.

\section{Discussion}

In 2003, Folpe and Weiss ${ }^{3}$ suggested that OFMT might belong to the group of translocation-associated sarcomas. They based their prediction mainly on the disparate lines of differentiation (schwannian and chondroid) displayed by the tumor cells and the lack of progressionrelated morphological changes in metastases compared with primary tumors. ${ }^{3}$ In the present study, we have shown that OFMT is recurrently associated with interchromosomal exchanges, resulting in rearrangement of a target gene in 6p21: PHF1. In one of the tumors, an in-frame EP400/PHF1 fusion transcript was found, whereas no fusion transcript was seen in the other two tumors.

Thus, OFMT is the second neoplasm, in addition to EST, in which PHF1 is involved in fusions with ectopic sequences. ESTs can be further subdivided into benign endometrial stromal nodules and the malignant variants endometrial stromal sarcoma and undifferentiated stromal sarcoma. The first fusion gene to be identified in EST was JAZF1/SUZ12, resulting from a $t(7 ; 17) .{ }^{14}$ Later, also, the JAZF1/PHF1 and EPC1/PHF1 chimeras, caused by a $\mathrm{t}(6 ; 7)$ and a $t(6 ; 10)$, respectively, were identified. ${ }^{10}$ Recently, high-grade ESTs were characterized by a t(10; 17), resulting in the YWHAE/FAM22 fusion gene. ${ }^{15}$ Chiang et $\mathrm{al}^{16}$ ascertained the relative frequencies of the three then known gene fusions (JAZF1/SUZ12, JAZF1) PHF1, and EPC1/PHF1) by using interphase FISH on sections from 81 ESTs. The most common fusion was JAZF1/SUZ12 (35\%), whereas JAZF1/PHF1 and EPC1/ PHF1 together accounted for $9 \%$ of the cases; the two PHF1 fusions were only identified in the malignant variants. Furthermore, rearrangement of the PHF1 locus without simultaneous rearrangement of the JAZF1 or EPC1 locus was found in an additional $9 \%$ of the cases, suggesting one or more alternate fusion partners in half of the cases with PHF1 involvement. ${ }^{16}$ Although OFMT and EST share no morphological or $\mathrm{IHC}$ features, they have in common the involvement of PHF1 in fusions with various $5^{\prime}$ partners. The involvement of the same gene in fusion genes in different tumor types is, however, not exclusive for OFMT and EST. For instance, the ETV6/NTRK3 fusion gene has been described in a variety of neoplasms, including leukemia, breast cancer, infantile fibrosarcoma, and mesoblastic nephroma of the kidney. Thus, the type of fusion and the cellular context in which it occurs are important for the phenotype of the tumor cells. ${ }^{17}$

The PHF1-associated chromosomal rearrangements are unusually complex in both tumor types. None of the three OFMTs from which metaphase spreads was available had a standard two-way translocation. Furthermore, at interphase FISH on sections from additional tumors, three of seven OFMTs with rearrangement of the PHF1 locus had loss of sequences upstream of the gene. The cytogenetic data on EST are still limited, but because of the transcriptional orientations of the genes, neither the
JAZF1/PHF1 nor the EPC1/PHF1 fusion can arise through a simple translocation. ${ }^{10}$

The human PHF1 gene is one of three human homologues of the polycomb-like $(P C l)$ gene in Drosophila melanogaster, and is known to interact with polycomb group proteins, important regulators of homeotic (Hox) genes in multicellular organisms. ${ }^{18,19}$ Specifically, PHF1 has interacted with the polycomb-repressive complex 2 (PRC2), which is essential for silencing of Hox genes and other genes regulating development. ${ }^{18-21}$ The PRC2 consists of several proteins, including SUZ12 (the alternative 3'partner in EST-associated chimeric proteins), EZH1, and $\mathrm{EZH} 2$, with the latter two constituting the enzymatic subunits responsible for the dimethylation or trimethylation of lysine 27 of histone $\mathrm{H} 3 .{ }^{20} \mathrm{PHF} 1$ also participated in the cellular response to DNA damage, suggesting that it might be involved in the repair of DNA double-stranded breaks. ${ }^{22} \mathrm{It}$, thus, seems reasonable to assume that the PHF1 rearrangements in the cell type(s) from which OFMT arises will affect a variety of biological processes.

A subset of tumors, especially those classified as malignant, lacked rearrangement of the PHF1 locus, indicating that OFMT is genetically heterogeneous. Graham et $\mathrm{al}^{6}$ recently suggested that OFMT develops primarily through inactivation of the SMARCB1 (also known as INI-1 or SNF5) gene in chromosome band 22q11. They based their hypothesis on the frequent $(75 \%$ of the cases) finding of weak or absent immunostaining for SMARCB1 in a series of 46 OFMTs, in combination with hemizygous loss of chromosome 22 in 5 of 7 cases at interphase $\mathrm{FISH} .{ }^{6}$ SMARCB1 is a core subunit of all known variants of the SWI/SNF chromatin remodeling complexes, which are multimeric ATP-dependent regulators of gene expression programs. ${ }^{23}$ SMARCB1 has a tumor repressor role (eg, by repressing the hedgehog and retinoblastoma pathways), and functional inactivation of SMARCB1 plays an important pathogenetic role in several tumor types. The prototypic example is malignant rhabdoid tumor, in which homozygous deletions or various other mutations of SMARCB1 are seen in close to $100 \%$ of the cases. ${ }^{23,24}$ In particular, there is an antagonistic relationship between SWI/SNF complexes and polycomb group proteins, ${ }^{23,25}$ and an attractive hypothesis would be that the underlying pathogenetic mechanism in most OFMTs is increased PRC2 activity, achieved either through deregulation of PHF1 or by inactivation of SMARCB1.

Although we detected frequent loss of one chromosome 22 at interphase FISH analysis of malignant OFMTs, reduced or absent immunostaining for SMARCB1 was not observed in any case. The reason for the discrepancy between our results and those of Graham et $\mathrm{al}^{6}$ is unclear, particularly because the same antibody and comparable techniques were used, albeit we used the antibody in more dilute form. For several reasons, we believe that other target genes on chromosome 22 should be considered. First, we, and Graham et al ${ }^{6}$ consistently, and by using different FISH probes, found signal patterns suggesting loss of the entire chromosome 22. Hence, the SMARCB1 locus is not specifically targeted. Second, although we cannot exclude inactivating point mutations in 
the remaining allele, loss of one copy of SMARCB1 is not sufficient for functional inactivation. ${ }^{23,24}$ Third, there are several other well-known tumor suppressor genes, such as NF2, that map to chromosome 22. Finally, the only malignant OFMT with an abnormal karyotype that has been reported so far $^{8}$ showed a complex karyotype including -22 . Thus, loss of one copy of chromosome 22 might be just one of several recurrent chromosomal imbalances in malignant OFMTs.

In one of the three tumors (patient 6) that could be studied in detail, a partner gene to PHF1 (ie, EP400) could be identified. EP400, which is also involved in the dynamics of chromatin structure, encodes an evolutionarily conserved ATPase of the SWI/SNF family. The EP400 protein interacts with several other proteins, including helicases and a histone acetyl transferase, mediating exchanges of different variants of histone H2A. In this capacity, it is involved in different cellular processes, such as regulation of gene transcription and DNA double-stranded break repair. Depending on cellular context, EP400 can activate or repress gene transcription through incorporation of the H2A variant, H2A.Z. ${ }^{26}$ In cases 1 and 11, the absence of a fusion transcript at $5^{\prime}$-RACE PCR and the lack of known coding sequences in the chromosomal segment attached to the $5^{\prime}$-side of PHF1 in patient 1 suggest that the important outcome of the chromosomal exchanges in OFMT is loss of $5^{\prime}$-regulatory sequences. This impression is reinforced by the extensive variation in potential $5^{\prime}$-partners, and by the fact that the entire coding region is included in all hitherto known PHF1 chimeras. However, the effects of structural alterations of the PHF1 protein should also be taken into account; the fusions described so far have been in frame, suggesting that chimeric proteins are expressed. Furthermore, the reported 5'-partners are functionally related in that their wild-type proteins all have been implicated in chromatin structure or DNA accessibility.

In summary, the findings presented herein strongly indicate that the pathogenetic basis of OFMT frequently involves rearrangement of the PHF1 gene, suggesting that epigenetic deregulation of PRC2 target genes is crucial for tumor development. From a practical, clinical point of view, our results suggest that FISH analysis for rearrangement of the PHF1 locus could serve as an excellent diagnostic tool, especially to identify OFMT cases with typical and atypical morphological characteristics. Suspected atypical and malignant cases could also be analyzed with regard to the status of chromosome 22, hemizygous loss being common in this subset. Furthermore, the results of our study clearly support the view that malignant OFMTs exist. PHF1 rearrangements were found in all subsets, although they were more common among typical and atypical cases. Of particular interest, case 1 , classified as a typical OFMT, had almost identical karyotypes in a local recurrence and a metastasis, showing beyond doubt that typical OFMTs can metastasize. Finally, it might be worthwhile to look for mutations/deletions of tumor suppressor genes mapping to chromosome 22 in atypical and malignant OFMTs that are negative for PHF1 rearrangements.

\section{References}

1. Enzinger FM, Weiss SW, Liang CY: Ossifying fibromyxoid tumor of soft parts: a clinicopathologic analysis of 59 cases. Am J Surg Pathol 1989, 13:817-827

2. Fisher $C$, Hedges M, Weiss SW: Ossifying fibromyxoid tumor of soft parts with stromal cyst formation and ribosome-lamella complexes. Ultrastruct Pathol 1994, 18:593-600

3. Folpe AL, Weiss SW: Ossifying fibromyxoid tumor of soft parts: a clinicopathologic study of 70 cases with emphasis on atypical and malignant variants. Am J Surg Pathol 2003, 27:421-431

4. Kilpatrick SE, Ward WG, Mozes M, Miettinen M, Fukunaga M, Fletcher $\mathrm{CD}$ : Atypical and malignant variants of ossifying fibromyxoid tumor: clinicopathologic analysis of six cases. Am J Surg Pathol 1995, 19 : 1039-1046

5. Miettinen M, Finnell V, Fetsch JF: Ossifying fibromyxoid tumor of soft parts: a clinicopathologic and immunohistochemical study of 104 cases with long-term follow-up and a critical review of the literature. Am J Surg Pathol 2008, 32:996-1005

6. Graham RP, Dry S, Li X, Binder S, Bahrami A, Raimondi SC, Dogan A, Chakraborty S, Souchek JJ, Folpe AL: Ossifying fibromyxoid tumor of soft parts: a clinicopathologic, proteomic, and genomic study. Am J Surg Pathol 2011, 35:1615-1625

7. Sovani V, Velagaleti GVN, Filipowicz E, Gatalica Z, Knisely AS: Ossifying fibromyxoid tumor of soft parts: report of a case with novel cytogenetic findings. Cancer Genet Cytogenet 2001, 127:1-6

8. Nishio J, Iwasaki H, Ohjimi $Y$, Ishiguro M, Isayama T, Naito M, Okabayashi $\mathrm{H}$, Kaneko $\mathrm{Y}$, Kikuchi M: Ossifying fibromyxoid tumor of soft parts: cytogenetic findings. Cancer Genet Cytogenet 2002, 133:124128

9. Kawashima H, Ogose A, Umezu H, Hotta T, Tohyama T, Tsuchiya $M$, Endo N: Ossifying fibromyxoid tumor of soft parts with clonal chromosomal aberrations. Cancer Genet Cytogenet 2007, 176: $156-160$

10. Micci F, Panagopoulos I, Bjerkehagen B, Heim S: Consistent rearrangement of chromosomal band 6 p21 with generation of fusion genes JAZF1/PHF1 and EPC1/PHF1 in endometrial stromal sarcoma. Cancer Res 2006, 66:107-112

11. Mandahl N, Heim S, Arheden K, Rydholm A, Willén H, Mitelman F: Three major cytogenetic subgroups can be identified among chromosomally abnormal solitary lipomas. Hum Genet 1988, 79:203208

12. Shaffer LG, Slovak ML, Campbell LJ: An International System for Human Cytogenetic Nomenclature (2009). Basel, Karger, 2009

13. Jin Y, Möller E, Nord KH, Mandahl N, Vult Von Steyern F, Domanski HA, Mariño-Enríquez A, Magnusson L, Nilsson J, Sciot R, Fletcher CDM, Debiec-Rychter M, Mertens F: Fusion of the AHRR and NCOA2 genes through a recurrent translocation $\mathrm{t}(5 ; 8)(\mathrm{p} 15 ; \mathrm{q} 13)$ in soft tissue angiofibroma results in upregulation of aryl hydrocarbon receptor target genes. Genes Chromosomes Cancer 2012, 51:510-520

14. Koontz JI, Soreng AL, Nucci M, Kuo FC, Pauwels P, van Den Berghe $\mathrm{H}$, Dal Cin P, Fletcher JA, Sklar J: Frequent fusion of the JAZF1 and JJAZ1 genes in endometrial stromal tumors. Proc Natl Acad Sci U S A 2001, 98:6348-6353

15. Lee C-H, Ou W-B, Mariño-Enriquez A, Zhu M, Mayeda M, Wang $Y$, Guo X, Brunner AL, Amant F, French CA, West RB, McAlpine JN, Gilks CB, Yaffe MB, Prentice LM, McPherson A, Jones SJM, Marra MA, Shah SP, van de Rijn M, Huntsman DG, Dal Cin P, DebiecRychter M, Nucci MR, Fletcher JA: 14-3-3 Fusion oncogenes in high-grade endometrial stromal sarcoma. Proc Natl Acad Sci U S A 2012, 109:929-934

16. Chiang S, Ali R, Melnyk N, McAlpine JN, Huntsman DG, Gilks CB, Lee $\mathrm{CH}$, Oliva E: Frequency of known gene rearrangements in endometrial stromal tumors. Am J Surg Pathol 2011, 35:1364-1372

17. Mitelman F, Johansson B, Mertens F: The impact of translocations and gene fusions on cancer causation. Nat Rev Cancer 2007, 7:233245

18. Cao R, Wang $\mathrm{H}, \mathrm{He} \mathrm{J}$, Erdiument-Bromage $\mathrm{H}$, Tempst $\mathrm{P}$, Zhang $\mathrm{Y}$ : Role of hPHF1 in H3K27 methylation and Hox gene silencing. Mol Cell Biol 2008, 28:1862-1872

19. Sarma K, Margueron R, Ivanov A, Pirrotta V, Reinberg D: Ezh2 requires PHF1 to efficiently catalyze $\mathrm{H} 3$ lysine 27 trimethylation in vivo. Mol Cell Biol 2008, 28:2718-2731 
20. Margueron R, Reinberg D: The Polycomb complex PRC2 and its mark on life. Nature 2011, 469:343-349

21. Richly $\mathrm{H}$, Aloia L, Di Croce L: Roles of the Polycomb group proteins in stem cells and cancer. Cell Death Dis 2011, 2:e204

22. Hong Z, Jiang J, Lan L, Nakajima S, Kanno S, Koseki H, Yasui A: A polycomb group protein, PHF1, is involved in the response to DNA double-strand breaks in human cell. Nucleic Acids Res 2008, 36: 2939-2947

23. Wilson BG, Roberts CWM: SWI/SNF nucleosome remodellers and cancer. Nat Rev Cancer 2011, 11:481-492
24. Roberts CWM, Biegel JA: The role of SMARCB1/INI1 in development of rhabdoid tumor. Cancer Biol Ther 2009, 8:412-416

25. Wilson BG, Wang X, Shen X, McKenna ES, Lemieux ME, Cho YJ, Koellhoffer EC, Pomeroy SL, Orkin SH, Roberts CWM: Epigenetic antagonism between polycomb and SWI/SNF complexes during oncogenic transformation. Cancer Cell 2010, 18:316-328

26. Mattera L, Courilleau C, Legube G, Ueda T, Fukunaga R, ChevillardBriet M, Canitrot Y, Escaffit F, Trouche T: The E1A-associated p400 protein modulates cell fate decisions by the regulation of ROS homeostasis. PLoS Genet 2010, 6:e1000983 